\title{
The Comparison Between Two Mathematical Contractile Elements Integrated into an hiPSC-CM In-silico Model
}

\author{
Mohamadamin Forouzandehmehr ${ }^{1}$, Nicolò Cogno ${ }^{2}$, Jussi T Koivumäki ${ }^{1}$, Jari Hyttinen ${ }^{1}$, Michelangelo \\ Paci $^{1}$ \\ ${ }^{1}$ Faculty of Medicine and Health Technology, Tampere University, Tampere, Finland \\ ${ }^{2}$ Institut für Festkörperphysik, Technische Universität Darmstadt, Darmstadt, Germany
}

\begin{abstract}
Human induced pluripotent stem cell-derived cardiomyocytes (hiPSC-CMs) are a valuable tool for in vitro drug testing and disease studies. As contractility has become one of the main experimental outputs, hiPSCCMs in silico models should also feature the mechanisms of force generation. Thus, we integrated two contractile elements (CE), Rice2008 and Negroni2015, into Paci2020 hiPSC-CM model. The simulated force- $\mathrm{Ca}^{2+}$ relationships from skinned versions of the CEs revealed rather close $p \mathrm{Ca}_{50}$ values for both CEs: 6.17 and 6.10, respectively for Rice 2008 and Negroni2015. However, Hill's coefficients for the two curves were 7.30 and 3.6. The relationships agreed with in vitro data from human engineered heart tissues. Most of the biomarkers measured from simulated spontaneous action potentials $(A P s)$ and $\mathrm{Ca}^{2+}$ transients (CaTs) showed good agreement with in vitro data for both CEs. The active peak force observed in paced conditions $(1 \mathrm{~Hz})$ and at extracellular $\mathrm{Ca}^{2+}$ concentration $\left(\left[\mathrm{Ca}^{2+}\right]_{o}\right.$ ) of $1.8 \mathrm{mM}$ was $0.011 \mathrm{mN} / \mathrm{mm}^{2}$ for Paci2020+Rice 2008 and $0.57 \mathrm{mN} / \mathrm{mm}^{2}$ for Paci2020+Negroni2015. These values match, qualitatively with the $0.26 \mathrm{mN} / \mathrm{mm}^{2}$ peak force reported previously in vitro at $\left[\mathrm{Ca}^{2+}\right]_{0}=1.8 \mathrm{mM}$. Our results set an opening to develop more sophisticated hiPSC-CM models featuring both electrophysiology and biomechanics.
\end{abstract}

\section{Introduction}

The role of computer-based modeling in cardiac pathophysiology investigations in a variety of scopes (from the arterial network to cell electrophysiological in silico models) through different approaches (finite element analysis to machine learning algorithm), has become increasingly important in recent years [1]-[7].

As a valuable modeling tool, human induced pluripotent stem cell-derived cardiomyocytes (hiPSC$\mathrm{CM}$ ) have been considered a promising structure for drug testing and disease studies, since they retain the same genetic information as the donor. Notably, the need for prediction of drug effects and cardiotoxicity highlights the importance of developing in silico methods as well as use of hiPSC-CMs; the fact which also has made it essentially supported by the Comprehensive In Vitro Proarrhythmia Assay (CiPA) initiative [8]-[10].

Biomarkers calculated from $\mathrm{Ca}^{2+}$ transients $(\mathrm{CaTs})$ are as valuable as the ones measured from action potential (AP), due to the vital role of $\mathrm{Ca}^{2+}$ cycling in cell functions and the increasing access to hiPSC-CM Ca ${ }^{2+}$ data. Notably, $\mathrm{Ca}^{2+}$ is fundamental in the heart excitationcontraction (EC) coupling, i.e., how the electrical and the mechanical properties of the heart are linked together and how the AP leads to the cardiomyocyte contraction. Indeed, the association of cardiac electrical and mechanical properties is shaped in view of $\mathrm{Ca}^{2+}$ cycling. Controversially, in previously developed cardiac muscle mechanical models, the contractile part, in effect, has been divorced from the electrophysiology formalism [11]. While this gap has started to be filled by a number of studies which have addressed the length and forcedependent relationships of $\mathrm{Ca}^{2+}$ and crossbridge construction, yet the majority has not gone beyond the cardiomyocyte ion handling and electrophysiological indices [12].

Improvement in understanding of the EC coupling, as the key player in generating sequential contraction of cardiac muscles, and the pro-arrhythmic risk assessments, highlight the need for a comprehensive mathematical hiPSC-CM model, specifically in mechanical terms. Here, two well-established mathematical contractile element (CE) models, namely Rice2008 [13] and Negroni2015 [14], have been integrated into the recently published Paci2020 hiPSC-CM in silico model [15]. We assessed the CE's impact on hiPSC-CM electrophysiology in terms of $\mathrm{AP}$ and $\mathrm{CaT}$ biomarkers and proposed a preliminary comparison of the capabilities of the two CEs in recapitulating in vitro hiPSC-CM force- $\mathrm{Ca}^{2+}$ data. As future steps, it is noteworthy that such findings can be validated against novel in vitro measurements reported previously on hiPSC-CMs [16]. 


\section{Materials and methods}

\subsection{Contractile element and integration}

In Negroni2015 machinery, myosin heads attach to actin in the overlapping area of thin and thick filaments. This zone emerges following their sliding past each other and enables cross bridges (XB) to form and as a result generates the force. XBs are assumed as elastic components which have a mobile end and a fixed end secured to the free end of thick filament (Fig.1).

Similarly, in Rice2008 CE structure, the action of cycling XBs is responsible for the development of the active force. Explicitly, the fraction of myosin heads in the overlap zone initiates the force development. However, the contributions of the passive force and the viscoelastic component must be considered to simulate a more comprehensive myofilament response. In our simulations, the muscle length of $2.1 \mu \mathrm{m}$ was assumed as the rest length at which there is no passive force. The optional series elastic element in Rice2008 is suitable for simulation of protocols which the internal sarcomere can shorten owing to the stretch in the compliant end. Also, we assumed the myofilament in the Rice $\mathrm{CE}$ has a Newtonian viscosity set to $0.3 \%$ of $\mathrm{F}_{\max } \mu \mathrm{m}^{-1} \mathrm{~s}^{-1}$ based on an experimental mean value [17].

The $\mathrm{Ca}^{2+}$ binding to troponin system is the main conception underlying the two CEs which were integrated to Paci2020 model. While the two CEs significantly differ from how they handle the states of troponin system, the crossbridge mechanism, and the $\mathrm{Ca}^{2+}$ bindings, both use the cytosolic $\mathrm{Ca}^{2+}$ to run their multiple states of troponin regulations and simulate the dynamic flux of $\mathrm{Ca}^{2+}$ towards the myofilament as the feedback. The detail of $\mathrm{Ca}^{2+}$ binding to troponin can be found in [13], [14].

\section{Results and discussion}

\subsection{Force-Ca $\mathrm{Ca}^{2+}$ relationship}

The simulated force- $\mathrm{Ca}^{2+}$ relationships showed a rather close pCa50 values for both CEs: 6.17 and 6.10 for Rice2008 and Negroni2015, respectively. Whereas, Hill's coefficients for the two curves were 7.30 and 3.60. As can be seen in Fig. 2, the relationships were in qualitative agreement with in vitro data obtained from human engineered heart tissues [18].

\subsection{Force development and $\mathrm{Ca}^{2+}$ transients after integration}

The active peak force observed in paced conditions (1 $\mathrm{Hz})$ and at extracellular $\mathrm{Ca}^{2+}$ concentration $\left(\left[\mathrm{Ca}^{2+}\right]_{0}\right)$ of $1.8 \mathrm{mM}$ was $0.011 \mathrm{mN} / \mathrm{mm}^{2}$ for Paci2020+Rice2008 and $0.57 \mathrm{mN} / \mathrm{mm}^{2}$ for Paci2020+Negroni2015. As can be observed also in Fig. 3, these values match, qualitatively, the $0.26 \mathrm{mN} / \mathrm{mm}^{2}$ the in vitro peak force reported previously by Stoehr et al. at $\left[\mathrm{Ca}^{2+}\right]_{0}=1.8 \mathrm{mM}[18]$.
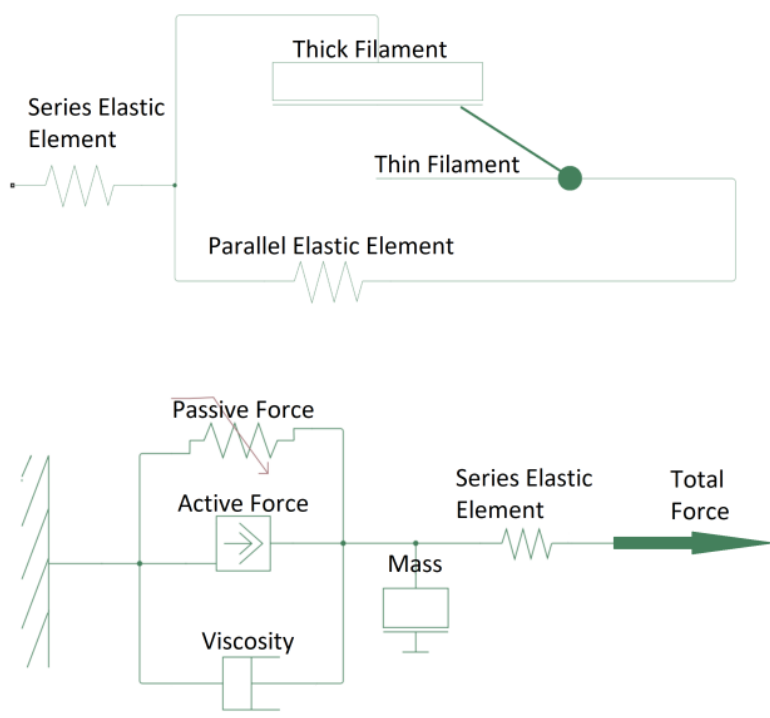

Figure 1. The mechanical schematic illustrations of the Negroni2015 (upper panel) and Rice2008 (lower panel).

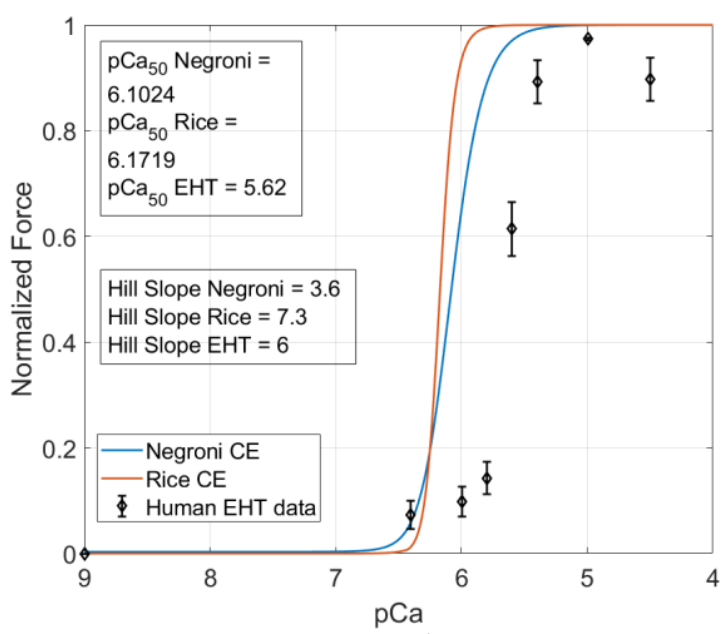

Figure 2. Simulated force- $\mathrm{Ca}^{2+}$ relationships of skinned versions of the Negroni model (blue), Rice model (orange), and recorded on engineered heart tissues (EHT) (in vitro data from [18]).

\subsection{Force-SL relationship}

As the developed force is directly influenced by the Sarcomere Length (SL), we studied the effect of change in SL on the developed active force in $1 \mathrm{~Hz}$ paced condition (Fig. 4). As can be seen, the increase in SL resulted in the elevation of peak force. Furthermore, the trend of simulated results follows its corresponding in vitro data reported for cat trabeculae [19]. 

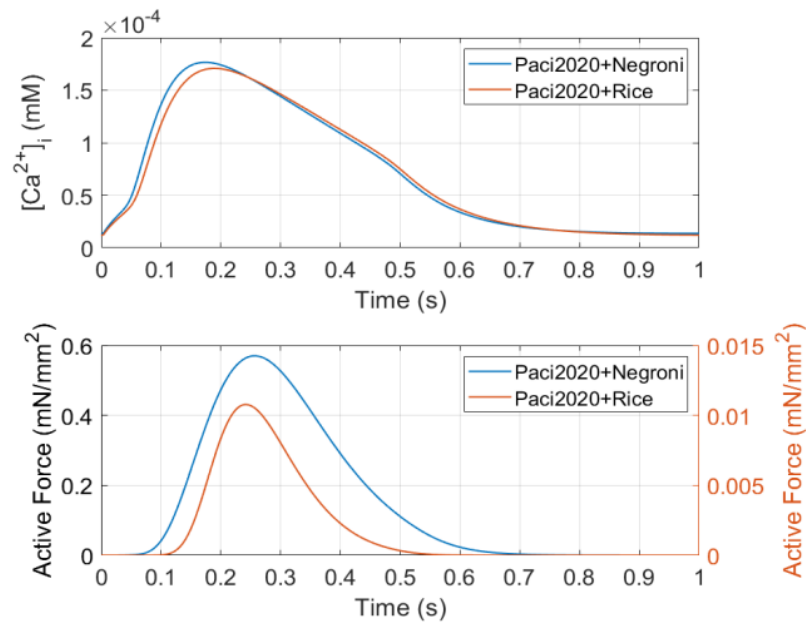

Figure 3. CaTs (upper panel) and the developed active force (lower panel) simulated for the Paci2020+Rice2008 and Paci2020+Negroni2015 models for $\left[\mathrm{Ca}^{2+}\right]_{\mathrm{o}}=1.8 \mathrm{mM}$ and $1 \mathrm{~Hz}$ pacing.
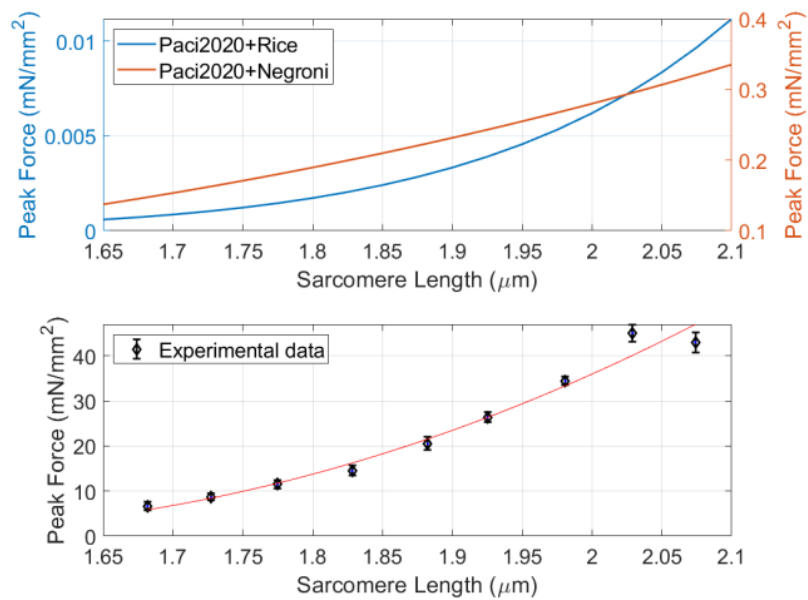

Figure 4. Calculated developed peak force of the two integrated hiPSC-CM model with respect to change in Sarcomere Length (SL) (upper panel). In vitro data of force-SL relationship measured in cat cardiac trabeculae from [19] (lower panel).

\subsection{Evaluation of biomarkers}

The ability to replicate key AP and CaT biomarkers was studied for the two integrated models. These simulations were done in spontaneous beating condition. In both hiPSC-CM models with integrated CEs, most of the biomarkers measured from simulated spontaneous APs and CaTs showed good agreement with the corresponding in vitro data. Notably, unlike the Paci2020+Negroni2015, the model with the Rice2008 CE replicated also the AP triangulation factor (AP Tri) of ventricular like hiPSC-CMs within the experimental range. Tables 1 and 2 show the biomarkers values for each model and indicate whether they have successfully recapitulated experimental results.
Table 1. Biomarker evaluation for Paci2020+Negroni2015. WER: within experimental range.

\begin{tabular}{|c|c|c|c|}
\hline Biomarker & $\begin{array}{l}\text { Simulated } \\
\text { Value }\end{array}$ & $\begin{array}{l}\text { Exp. Value } \\
\text { (Mean } \pm \text { SD) }\end{array}$ & WER \\
\hline APA (mV) & 104.6 & $104 \pm 6$ & true \\
\hline $\operatorname{MDP}(\mathrm{mV})$ & -75.3 & $-75.6 \pm 6.6$ & true \\
\hline AP CL (ms) & 1504.9 & $1700 \pm 548$ & true \\
\hline $\mathrm{dV} / \mathrm{dt} \max (\mathrm{V} / \mathrm{s})$ & 13.5 & $27.8 \pm 26.3$ & true \\
\hline $\mathrm{APD}_{10}(\mathrm{~ms})$ & 92.3 & $74.1 \pm 26.3$ & true \\
\hline $\mathrm{APD}_{30}(\mathrm{~ms})$ & 230.7 & $180 \pm 59$ & true \\
\hline $\mathrm{APD}_{90}(\mathrm{~ms})$ & 377.3 & $415 \pm 119$ & true \\
\hline AP Tri & 3.69 & $2.5 \pm 1.1$ & false \\
\hline CaT DURATION (ms) & 641.3 & $805 \pm 188$ & true \\
\hline $\mathrm{CaT}_{\text {tRise }}{ }_{10, \text { peak }}(\mathrm{ms})$ & 131.4 & $270 \pm 108$ & false \\
\hline Cat tRise $_{10,50}(\mathrm{~ms})$ & 38.3 & $82.9 \pm 50.5$ & true \\
\hline $\mathrm{CaT}_{\text {tRise }} 0,90(\mathrm{~ms})$ & 82.4 & $167 \pm 70$ & false \\
\hline CaT tDecay90,10 (ms) & 331 & $410 \pm 100$ & true \\
\hline
\end{tabular}

Table 2. Biomarker evaluation for Paci2020+Rice2008.

\begin{tabular}{|c|c|c|c|}
\hline Biomarker & $\begin{array}{l}\text { Simulated } \\
\text { Value }\end{array}$ & $\begin{array}{l}\text { Exp.Value } \\
(\text { Mean } \pm \text { SD) }\end{array}$ & WER \\
\hline APA $(\mathrm{mV})$ & 104.7 & $104 \pm 6$ & true \\
\hline $\operatorname{MDP}(\mathrm{mV})$ & -75.3 & $-75.6 \pm 6.6$ & true \\
\hline AP CL (ms) & 1558.6 & $1700 \pm 548$ & true \\
\hline $\mathrm{dV} / \mathrm{dt} \max (\mathrm{V} / \mathrm{s})$ & 14.0 & $27.8 \pm 26.3$ & true \\
\hline $\mathrm{APD}_{10}(\mathrm{~ms})$ & 93.4 & $74.1 \pm 26.3$ & true \\
\hline $\mathrm{APD}_{30}(\mathrm{~ms})$ & 234.5 & $180 \pm 59$ & true \\
\hline $\mathrm{APD}_{90}(\mathrm{~ms})$ & 384.9 & $415 \pm 119$ & true \\
\hline AP Tri & 3.57 & $2.5 \pm 1.1$ & true \\
\hline CaT DURATION (ms) & 680.4 & $805 \pm 188$ & true \\
\hline CaT tRise 10, peak $(\mathrm{ms})$ & 136.3 & $270 \pm 108$ & false \\
\hline Cat tRise ${ }_{10,50}(\mathrm{~ms})$ & 39.4 & $82.9 \pm 50.5$ & true \\
\hline CaT tRise $_{10,90}(\mathrm{~ms})$ & 86.2 & $167 \pm 70$ & false \\
\hline CaT tDecay $90,10(\mathrm{~ms})$ & 348.7 & $410 \pm 100$ & true \\
\hline
\end{tabular}

To elucidate, AP biomarkers assessed are listed as: APA (AP amplitude), MDP (maximum diastolic potential), CL (cycle length), dV/dt max (maximum upstroke velocity), $\mathrm{APD}_{10}$ and $\mathrm{APD}_{30}$ and $\mathrm{APD}_{90}(\mathrm{AP}$ duration at 10,30,90\% of repolarization), AP Tri (AP triangulation factor). Also, $\mathrm{CaT}$ biomarkers are DURATION (duration of the transient), tRise 10 , peak (time to peak), tRise ${ }_{10,50}$ and tRise ${ }_{10,90}$ (rise time from 10 to $50 \%$ and $90 \%$ of maximum threshold), and tDecay ${ }_{90,10}$ (decay time from 90 to $10 \%$ ).

\section{Conclusion}

The role of hiPSC-CMs and their mathematical modelling, has become increasingly prevalent in fundamental studies of electrophysiological and contractile function, as well as, pharmacological tests. 
Here, two established CE models, namely Rice2008 and Negroni2015, have been integrated into Paci2020 hiPSCCM model. Our work represents a first attempt to move beyond electrophysiology in in silico descriptions of hiPSC-CMs, through evaluating two of CE models. Our results show a qualitative agreement with in vitro data from hiPSC-CMs and represent a starting point to develop more refined hiPSC-CM models combining both electrophysiology and contractility. This new generation of in silico models will be able to simulate the effects of diseases affecting not only the electrophysiology (e.g. channelopathies), but also the contractile machinery (e.g. hypertrophic or dilated cardiomyopathy).

\section{Acknowledgments}

Mohamadamin Forouzandehmehr was supported by Faculty of Medicine and Health Technology, Tampere University. Dr. Michelangelo Paci was supported by the Academy of Finland (project CardSiPop, decision number 307967). Dr. Koivumäki was supported by Academy of Finland Centre of Excellence in Body-on-Chip Research and Pirkanmaa regional fund of the Finnish Cultural Foundation (grant numbers 50171514 and 50201322).

\section{References}

[1] M. Forouzandehmehr and A. Shamloo, "Margination and adhesion of micro- and nanoparticles in the coronary circulation: A step towards optimised drug carrier design," Biomech. Model. Mechanobiol., 2018.

[2] A. Shamloo, A. Amani, M. Forouzandehmehr, and I. Ghoytasi, "In silico study of patient-specific magnetic drug targeting for a coronary LAD atherosclerotic plaque," Int. J. Pharm., 2019.

[3] E. Passini et al., "Human in silico drug trials demonstrate higher accuracy than animal models in predicting clinical pro-arrhythmic cardiotoxicity," Front. Physiol., 2017.

[4] M. C. Lancaster and E. A. Sobie, "Improved prediction of drug-induced torsades de pointes through simulations of dynamics and machine learning algorithms," Clin. Pharmacol. Ther., 2016.

[5] A. Shamloo and M. Forouzandehmehr, "Personalised deposition maps for micro- and nanoparticles targeting an atherosclerotic plaque: attributions to the receptormediated adsorption on the inflamed endothelial cells," Biomech. Model. Mechanobiol., 2019.

[6] D. Shah et al., "hiPSC-derived cardiomyocyte model of LQT2 syndrome derived from asymptomatic and symptomatic mutation carriers reproduces clinical differences in aggregates but not in single cells," Cells, 2020.

[7] J. Parikh, V. Gurev, and J. J. Rice, "Novel two-step classifier for Torsades de Pointes risk stratification from direct features," Front. Pharmacol., 2017.

[8] A. Muszkiewicz et al., "Variability in cardiac electrophysiology: Using experimentally-calibrated populations of models to move beyond the single virtual physiological human paradigm," Prog. Biophys.
Mol. Biol., 2016.

[9] O. J. Britton et al., "Experimentally calibrated population of models predicts and explains intersubject variability in cardiac cellular electrophysiology," Proc. Natl. Acad. Sci. U. S. A., 2013.

[10] D. G. Strauss et al., "Comprehensive In Vitro Proarrhythmia Assay (CiPA) Update from a Cardiac Safety Research Consortium / Health and Environmental Sciences Institute / FDA Meeting," Ther. Innov. Regul. Sci., 2019.

[11] L. Chin, P. Yue, J. J. Feng, and C. Y. Seow, "Mathematical simulation of muscle cross-bridge cycle and force-velocity relationship," Biophys. J., 2006.

[12] A. R. Soltis and J. J. Saucerman, "Synergy between CaMKII substrates and $\beta$-adrenergic signaling in regulation of cardiac myocyte $\mathrm{Ca} 2$ handling," Biophys. J., 2010.

[13] J. J. Rice, F. Wang, D. M. Bers, and P. P. De Tombe, "Approximate model of cooperative activation and crossbridge cycling in cardiac muscle using ordinary differential equations," Biophys. J., 2008.

[14] J. A. Negroni et al., " $\beta$-adrenergic effects on cardiac myofilaments and contraction in an integrated rabbit ventricular myocyte model," J. Mol. Cell. Cardiol., 2015.

[15] M. Paci et al., "All-Optical electrophysiology refines populations of in silico human iPSC-CMs for drug evaluation," Biophys. J., 2020.

[16] A. Ahola, R. P. Pölönen, K. Aalto-Setälä, and J. Hyttinen, "Simultaneous measurement of contraction and calcium transients in stem cell derived cardiomyocytes," Ann. Biomed. Eng., 2018.

[17] P. P. de Tombe and H. E. ter Keurs, "An internal viscous element limits unloaded velocity of sarcomere shortening in rat myocardium.," J. Physiol., 1992.

[18] A. Stoehr et al., "Automated analysis of contractile force and $\mathrm{Ca} 2+$ transients in engineered heart tissue," Am. J. Physiol. - Hear. Circ. Physiol., 2014.

[19] P. P. De Tombe and H. E. D. J. Ter Keurs, "Sarcomere dynamics in cat cardiac trabeculae," Circ. Res., 1991.

Address for correspondence:

Mohamadamin Forouzandehmehr.

Arvo Ylpön katu 34, (Arvo building, $2^{\text {nd }}$ floor), D224 33520 Tampere, Finland.

mohamadamin.forouzandehmehr@tuni.fi 